\title{
A Computational Model to Assess Poststenting Wall Stresses Dependence on Plaque Structure and Stenosis Severity in Coronary Artery
}

\author{
Zuned Hajiali, Mahsa Dabagh, and Payman Jalali \\ School of Technology, Lappeenranta University of Technology, 53851 Lappeenranta, Finland \\ Correspondence should be addressed to Mahsa Dabagh; mahsa@lut.fi
}

Received 17 December 2013; Revised 1 July 2014; Accepted 26 July 2014; Published 10 September 2014

Academic Editor: Yonghong Wu

Copyright (C) 2014 Zuned Hajiali et al. This is an open access article distributed under the Creative Commons Attribution License, which permits unrestricted use, distribution, and reproduction in any medium, provided the original work is properly cited.

The current study presents computational models to investigate the poststenting hemodynamic stresses and internal stresses over/within the diseased walls of coronary arteries which are in different states of atherosclerotic plaque. The finite element method is applied to build the axisymmetric models which include the plaque, arterial wall, and stent struts. The study takes into account the mechanical effects of the opening pressure and its association with the plaque severity and the morphology. The wall shear stresses and the von Mises stresses within the stented coronary arteries show their strong dependence on the plaque structure, particularly the fibrous cap thickness. Higher stresses occur in severely stenosed coronaries with a thinner fibrous cap. Large stress concentrations around the stent struts cause injury or damage to the vessel wall which is linked to the mechanism of restenosis. The in-stent restenosis rate is also highly dependent on the opening pressure, to the extent that stenosed artery is expanded, and geometry of the stent struts. The present study demonstrates, for the first time, that the restenosis is to be viewed as a consequence of biomechanical design of a stent repeating unit, the opening pressure, and the severity and morphology of the plaque.

\section{Introduction}

Atherosclerosis is one of the major causes of death worldwide. The coronary artery is the most subjected artery to atherosclerosis, where plaque is accumulated within the inner walls of the vessel [1]. In case of partial or total lumen obstruction, the stent implantation is the common procedure to resume the vascular lumen with minimal surgical complication $[1,2]$. However, the restenosis in the coronary artery remains a recurrent problem in $30 \%-40 \%$ of the cases after the patient has received a bare metal stent [3].

The mechanism of restenosis has been hypothesized to involve the injury or damage to the vessel wall due to the high stresses generated around the stent when it expands [4]. In vivo and in vitro studies have revealed that stent structure (stent configuration, its global length, the delivery system, the struts dimensions, shape, spacing, and many others) influences restenosis and thrombus formation between struts $[1,3,5]$. Several studies have investigated the stresses generated in the vascular wall as a way to compare the stents relative to their potential for restenosis $[4,6]$. A study of the arterial tissue within repeating units of different commercially available stent designs has been performed by Prendergast et al. [4]. They reported tissue prolapse and arterial wall stresses; however, the stent-artery contact was not simulated in that analysis. The most detailed numerical model of a cardiovascular intervention to date is the model developed by Holzapfel et al. [7]. They developed a threedimensional (3D) model using the finite-element method to determine the stresses induced within the vessel for a balloon angioplasty and stenting procedures. However, the localized stresses around the stent struts, that is, the stresses most likely to provoke in-stent restenosis, were not computed. Lally et al. [6] suggested that the two different stent designs have significantly different restenosis rates and confirmed the use of computer-based finite-element analysis as a preclinical 
testing methodology to analyze the biomechanical attributes of cardiovascular stents. On the other hand, the mechanical behavior of the stent and biomechanical interaction between the stent and the artery has been tied to the artery hemodynamics $[8,9]$. The hemodynamics is affected by the formation of vortices and stagnation which is induced by the parts of stent struts protruding into the lumen $[10,11]$.

Moreover, the magnitude and distribution of stresses within an atherosclerotic artery are affected by the blood pressure, the arterial wall thickness and properties, and the severity, structure, and properties of the plaque [12]. Gao and Long [13] investigated the influence of plaque structure on the stress distribution, taking into account combinations of fibrous cap and necrotic core volume but with the same degree of stenosis. They found that the stress levels are highly dependent on plaque structure and are much more sensitive to the changes in the fibrous thickness than the necrotic core volume. Ohayon et al. [14] demonstrated that the stability of plaque cannot be viewed as a consequence of fibrous cap alone but rather as a combination of cap thickness, necrotic core, and arterial remodeling index. Pericevic et al. [15] studied the influence of plaque structure and inflation pressure on wall stress during stent expansion. They studied the influence of plaque stiffness and stent deployment pressures, while the importance of stenosis severity in their study was ignored and the plaque was modeled as one layer. Numerous computational studies have combined the mechanical behavior of stents with the hemodynamic inside the arteries to correlate the stent design with the restenosis rates that may help to develop novel stent designs $[1,2$, $6,11,16,17]$. However, in these studies, the arterial wall and the plaque have been modeled as single layers, and the impact of stenosis severity and the multilayer wall and plaque composition in the reoccurrence of stenosis have been ignored.

In the present study, an attempt is made to implement a simple computational model for studying the effects of plaque severity and structure on surface and internal stresses of arterial wall in stented coronaries. Numerical simulations are carried out in small segments of locally straight human coronary artery consisting of multilayer plaque and arterial wall around a single stent strut. The wall stresses are calculated in different degrees of stenosis severity. Various pressures are applied to open the stenosed coronaries of different severities to the diameter of the healthy lumen. Besides, a fixed value of pressure is applied to open stenosed coronaries to see how stenosis rate affects the ability of artery to be opened.

\section{Materials and Methods}

Several axisymmetric models of stent-plaque-artery are generated to represent the stented coronary arteries at different stages of atherosclerotic lesion growth. Stress distribution is studied inside the plaque and arterial wall, as well as the shear stress over the luminal surface of stented artery. The models are generated and solved using the finite-element based package of COMSOL Multiphysics 3.5a.
2.1. Model Geometry. The stented human coronary artery is created in an axisymmetric geometry containing a ring-like stent [9] placed in a cylindrical unit of stenosed artery with the length of $1.25 \mathrm{~mm}$ [18], an internal diameter of $3.0 \mathrm{~mm}$, and a thickness of $0.4 \mathrm{~mm}$ [11], as shown in Figure 1(b). A 3D schematic view of the artery by the ring-like stent is demonstrated in Figure 1(a), where various layers of plaque and the outer media layer are also shown. An axisymmetric plane AX is applied to cut the artery because of the axisymmetric shape which is shown in Figure 1(b). The plaque is a multilayer medium with the thicknesses of $0.34 \mathrm{~mm}, 0.55 \mathrm{~mm}$, and $0.83 \mathrm{~mm}$ in the stenoses of $40 \%, 60 \%$, and $80 \%$, respectively. The plaque has three layers as fibrous cap, soft necrotic core, and fibrosis. It has been shown that the area fraction of necrotic core with respect to the total area of the plaque naturally increases with the stenosis degree [19]. Virmani et al. [20] measured the area fraction of necrotic core in different stages of plaque growth in human coronaries. Those data have been applied in the present study. Table 1 represents the area fraction of the necrotic core for $40 \%, 60 \%$, and $80 \%$ stenosed coronaries. Three different thicknesses of fibrous cap have been taken into account, namely, thin, medium, and thick (values given in Table 1). This will signify the role of fibrous cap thickness in the blood flow dynamics around the stent strut. The ring-like stent described above has a rectangular cross section with the thickness of $0.1 \mathrm{~mm}$ [1] and the width of $0.15 \mathrm{~mm}$ [11].

The stent struts are assumed to be repeated periodically, where only one periodic unit is considered in the model [9]. This assumption is made to save computational time because no significant difference is observed in the wall shear stress distribution when multiple struts are present in the model as it will be shown in the results. The stent strut is kept fixed while the plaque and arterial wall are constrained in the axial direction with permission to deform in radial direction. This condition prevents the tangential deformation of the stenosed artery [21] with no slip allowed between the strut and plaque contact face. The expansion of the stenosed arteries is performed as explained below.

The stent strut is not initially positioned in place. Internal pressure is increased to let the coronaries with $40 \%, 60 \%$, and $80 \%$ stenoses reach the healthy lumen diameter of $2.68 \mathrm{~mm}$. Table 2 shows the pressure values applied to the stenosed coronaries of the above-mentioned stenosis percentages. In the following step, the stent strut is located in place. The insertion of stent naturally boosts the internal stresses as well as external compression imposed by adjacent tissue [22]. In this context, various factors are involved including the geometry and mechanical properties of stent and arterial wall and neighboring tissue, wall deformation, and blood pressure. In the present study, an external pressure will be created over the outer surface of the artery due to the opening of stent. The internal lumen pressure is decreased to the mean blood pressure of $100 \mathrm{mmHg}$. The value of the external pressure is expressed relative to the internal lumen pressure of $100 \mathrm{mmHg}$ so that the net pressure difference applied to the outer surface of the stenosed artery remains $15 \mathrm{mmHg}$ $[22,23]$. This value has been assumed in all stenoses cases to eliminate the effect of its variation from the results. The 
TABLE 1: The area fraction of necrotic core with respect to the total plaque area, lumen diameter (LD), and the varying fibrous cap thickness in thin, medium, and thick sizes (corresponding to the degree of stenosis).

\begin{tabular}{lcccr}
\hline Degree of stenosis (\%) & Necrotic core (\%) & Initial (mm) \\
& & LD & Fibrous cap thickness \\
Medium & 0.132 & 0.197 \\
\hline 40 & 22 & 2.32 & 0.094 & 0.187 \\
60 & 32 & 1.90 & 0.120 & 0.240 \\
80 & 42 & 1.34 & 0.361 \\
\hline
\end{tabular}

TABLE 2: Pressure values, lumen diameter (LD), and fibrous cap thickness (FCT) for $40 \%, 60 \%$, and $80 \%$ stenosed arteries under two separate expansion schemes. In the left side, the pressure values required to open the stenosed artery to the healthy lumen diameter are given. In the right side, the lumen diameter of stenosed arteries is given when the arteries are expanded with a fixed pressure of $100 \mathrm{mmHg}$.

\begin{tabular}{|c|c|c|c|c|c|c|c|c|c|c|c|c|}
\hline \multirow{3}{*}{$\begin{array}{l}\text { Degree of } \\
\text { stenosis (\%) }\end{array}$} & \multicolumn{6}{|c|}{ Displaced to healthy lumen diameter of $2.68 \mathrm{~mm}$} & \multicolumn{6}{|c|}{ Displaced under fixed pressure of $100 \mathrm{mmHg}$} \\
\hline & \multicolumn{2}{|c|}{ Thin } & \multicolumn{2}{|c|}{ Medium } & \multicolumn{2}{|c|}{ Thick } & \multicolumn{2}{|c|}{ Thin } & \multicolumn{2}{|c|}{ Medium } & \multicolumn{2}{|c|}{ Thick } \\
\hline & Pressure & FCT & Pressure & FCT & Pressure & FCT & LD & FCT & LD & FCT & LD & FCT \\
\hline 40 & 134 & 0.058 & 140 & 0.115 & 146 & 0.173 & 2.59 & 0.058 & 2.58 & 0.120 & 2.57 & 0.181 \\
\hline 60 & 450 & 0.063 & 510 & 0.126 & 550 & 0.195 & 2.08 & 0.086 & 2.06 & 0.175 & 2.05 & 0.264 \\
\hline 80 & 1255 & 0.027 & 1620 & 0.069 & 1830 & 0.132 & 1.45 & 0.113 & 1.43 & 0.229 & 1.41 & 0.348 \\
\hline
\end{tabular}

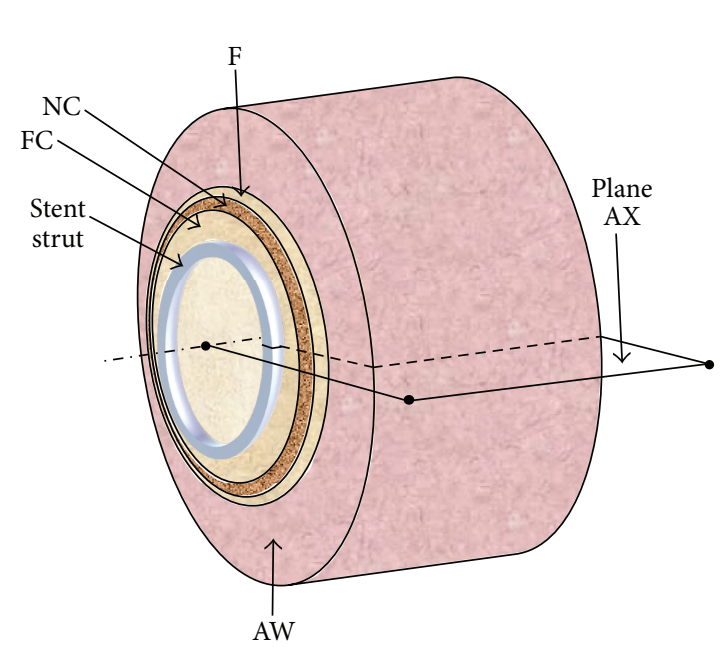

(a)

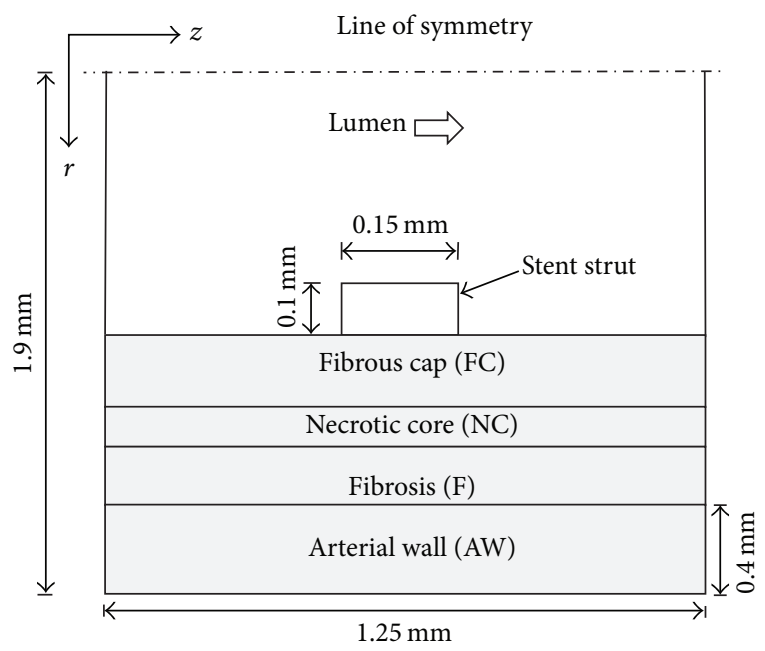

(b)

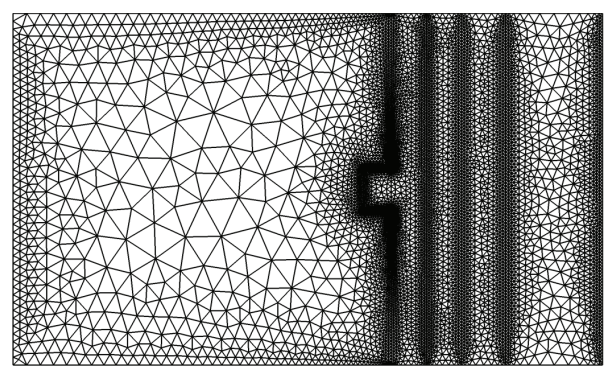

(c)

FIGURE 1: (a) 3D schematic view of stent strut placed in coronary artery with three distinctive plaque layers as fibrosis cap (FC), necrotic core (NC), and fibrosis (F) attached to arterial wall (AW). (b) Sectioning the artery with axisymmetric plane AX, showing the two-dimensional axisymmetric plane used in simulations. (c) Mesh grid used in simulations. 
TABLE 3: Constant values of parameters in the polynomials representing the inlet velocity waveform.

\begin{tabular}{lcccccccccccc}
\hline Parameter & $a_{1}$ & $a_{2}$ & $b_{1}$ & $b_{2}$ & $b_{3}$ & $b_{4}$ & $b_{5}$ & $c_{1}$ & $c_{2}$ & $d_{1}$ & $d_{2}$ & $d_{3}$ \\
\hline Constant value & -0.143 & 0.132 & -457.35 & 397.71 & -128.92 & 17.69 & -0.505 & 4.555 & -1.425 & 0.690 & -1.148 & 0.603 \\
\hline
\end{tabular}

plaque and arterial wall are allowed to deform under this value of external pressure, noting that the stent strut is kept fixed in the luminal side.

In addition to the above-mentioned cases, where different pressure values are applied for various stenoses to reach the healthy artery diameter, a benchmark case is created for each stenosis in which a constant internal pressure of $100 \mathrm{mmHg}$ is applied for the initial expansion of the artery. The diameter of the artery expands, under pressure of $100 \mathrm{mmHg}$, to $3.20 \mathrm{~mm}$, $3.10 \mathrm{~mm}$, and $3.04 \mathrm{~mm}$ corresponding to the stenoses of $40 \%$, $60 \%$, and $80 \%$. The rest of simulation procedure has been as explained for previous cases. Table 2 compares the values of lumen diameter and fibrous cap thickness under expansion with the fixed pressure of $100 \mathrm{mmHg}$ to those corresponding to the expansion with variable pressures.

2.2. Material Properties. The arterial wall is modeled as a viscoelastic layer that can be simulated by the generalized Maxwell model also found in COMSOL Multiphysics 3.5a $[7,24]$. The shear modulus for the arterial wall is taken as $G_{\text {wall }}=5.6 \mathrm{kPa}$ based on the data from Baldewsing et al. [25]. The arterial wall is assumed to be incompressible (i.e., Poisson's ration $=0.49)[2,6]$. Thus, the bulk modulus of the wall, $K_{\text {wall }}$, tends to infinity, taken as $10^{20} \mathrm{~Pa}$, in the present study. The three layers of the plaque are also assumed to be incompressible and represented by the generalized Maxwell model. The shear modulus of fibrous cap and fibrosis layers are taken as $200 \mathrm{kPa}$, whereas the soft necrotic core has the shear modulus of $G_{\text {core }}=3.3 \mathrm{kPa}[26]$. The density of arterial wall and plaque is considered as $\rho=1000 \mathrm{~kg} \cdot \mathrm{m}^{-3}$ [27]. The deformations of arterial wall and plaque layers take place statically.

2.3. Computational Method. The blood is modeled as a Newtonian and incompressible fluid with a density of $1060 \mathrm{~kg} \cdot \mathrm{m}^{-3}$ and a dynamic viscosity of $0.0035 \mathrm{~Pa} \cdot \mathrm{s}[9,11]$. Parabolic velocity profiles are applied, at the inlet, for steady and transient conditions, as

$$
u_{z}=u_{\max }\left(1-\frac{r^{2}}{r_{\text {in }}^{2}}\right)
$$

where $r_{\text {in }}$ is the radius at the inlet and $u_{\max }$ is the maximum velocity at the inlet for steady conditions as $0.35 \mathrm{~m} / \mathrm{s}$ [28]. For transient simulations $\left(u_{\max }\right.$ in $(1)$ is substituted by $u_{\max }(t)$ of (2)), a set of polynomials representing the time dependent inlet velocity for one cardiac cycle is used, given by (2) [29], where the constants are given in Table 3.

$$
\begin{aligned}
& u_{\max }(t) \\
& = \begin{cases}\left(a_{1} t+a_{2}\right), & 0 \leq t(s) \leq 0.054, \\
\left(b_{1} t^{4}+b_{2} t^{3}+b_{3} t^{2}+b_{4} t+b_{5}\right), & 0.054<t(s) \leq 0.341, \\
\left(c_{1} t+c_{2}\right), & 0.341<t(s) \leq 0.373, \\
\left(d_{1} t^{2}+d_{2} t+d_{3}\right), & 0.373<t(s) \leq 0.735 .\end{cases}
\end{aligned}
$$

Transient simulations are performed for three cardiac cycles, while each cardiac period is $0.735 \mathrm{~s}$ (Figure 5(a)). Since there was no significance difference between the last and second last cycles, the results of the last cycle will be presented in the following sections. At the outlet, a pressure with the value of $100 \mathrm{mmHg}$ is specified. The deformed structure only served as a boundary, where the flow simulations are carried out numerically by solving Navier-Stokes equations using the finite-element method, described by

$$
\begin{gathered}
\rho \frac{\partial u}{\partial t}+\rho(\mathbf{u} \cdot \nabla) \mathbf{u}=\nabla \cdot\left[-p \mathbf{I}+\eta\left(\nabla \mathbf{u}+(\nabla \mathbf{u})^{T}\right)\right], \\
\nabla \cdot \mathbf{u}=0
\end{gathered}
$$

where $\rho$ is density, $\eta$ is dynamic viscosity of blood, $\mathbf{u}$ is velocity vector, and $p$ is pressure. For steady simulations, the term $\rho \partial u / \partial t$ equals zero. Convergence criterion for the continuity and momentum residuals was kept at $10^{-6}$.

\section{Results and Discussion}

The computational results for the von Mises stress and wall shear stress were determined to be independent of mesh density. Computational mesh was employed for each layer consisting of 4283, 3467, 2150, 2170, and 2502 triangular elements in lumen, fibrosis cap, necrotic core, fibrosis, and arterial wall, respectively. Finer meshes were prescribed in the regions of interest near the strut and the walls of plaque and artery. Since the maximum value of von Mises stress in fibrous cap was sensitive to mesh changes, the mesh was additionally refined around the stent corners where stress concentration is very high. The model was solved using a generalized linear solver, namely, the UMFPACK solver. Simulations were performed on a Dell PRECISION T3600, 12 processor computers with $64 \mathrm{~GB}$ RAM. During the solution process for the entire domain of the lumen and stenosed arterial wall, there were approximately 90,000 degrees of freedom. Postprocessing results for the von Mises stress, wall shear 
stress, and flow profiles were obtained using postprocessing features of Comsol Multiphysics 3.5a package.

Following grid-independence analysis, the results of wall shear stress and von Mises stress are presented and discussed for the axisymmetric models of stent-plaque-artery, in which atherosclerotic plaque size and morphology are taken into account. Figure 2(a) shows the wall shear stress distribution on the stenosed arterial wall after insertion of the stent with multiple struts. A periodic distribution of wall shear stress is observed for $40 \%, 60 \%$, and $80 \%$ stenosed coronary arteries. However, the wall shear stress vary by $0.3 \mathrm{~Pa}$ from inlet to the first strut and $0.1 \mathrm{~Pa}$ from last strut to the exit of single and multiple strut models. In order to provide more evidence of periodicity, the velocity profiles are demonstrated at different locations along multiple struts in Figure 2(b). It clearly displays the periodic feature of the flow in the lumen in the units corresponding to consecutive struts. Moreover, the distribution of von Mises stress along the interfaces of various layers is plotted in Figures 2(c)-2(e) corresponding to the interfaces of fibrous cap-necrotic core, necrotic corefibrosis, and fibrosis-arterial wall, respectively. These figures indicate clearly the periodicity of stress in various units of struts. Therefore, the assumption of single strut is justified in the rest of the results presented here.

Figure 3 demonstrates the distribution of the wall shear stress on the stented arterial wall surface in various strut thicknesses which have been employed by Balossino et al. [1] and Jiménez and Davies [9]. Thinner struts increase wall shear stress magnitude, as shown in Figures 3(a), 3(b), and 3 (c). The length of recirculation zone in both sides of the strut reduces with decreasing the strut thickness for all percentages of stenosis. The results are in good agreement with the data reported by Jiménez and Davies [9].

3.1. Wall Shear Stress Distribution. The stenosed coronary arteries with $40 \%, 60 \%$, and $80 \%$ stenoses are pressurized to reach the lumen diameter of $2.68 \mathrm{~mm}$ which corresponds to the healthy lumen. Prior to the pressurization of the model artery, the blood flow simulation was performed within the stenosed arteries with $40 \%, 60 \%$, and $80 \%$ stenoses, which resulted in the values of wall shear stress as $2.1 \mathrm{~Pa}, 2.6 \mathrm{~Pa}$, and 3.6 Pa, respectively. This was obtained using the parabolic inlet velocity profile as described before, while the diameters of stenosed arteries were different.

Flow simulations were performed after the insertion of stent strut (described in Section 2). Figure 4(a) shows the distribution of wall shear stress along the axial direction of the stented coronary artery in different stenoses. Note that Figure 4(a) corresponds to the stenosis with the medium fibrous cap thickness of $0.187 \mathrm{~mm}$ (given in Table 1). In Figure 4(a), the straight line from $z=0.55$ to $z=0.7 \mathrm{~mm}$ represents the location of the strut. Figure 4(a) reveals that the wall shear stress decreases from its maximum at the inlet (the middle of two consecutive struts) to values below $0.5 \mathrm{~Pa}$ in the upstream vicinity of the strut. In the downstream vicinity of the strut, the value of wall shear stress is below $0.5 \mathrm{~Pa}$ and it rises along the deformed zone to a plateau at $1.25 \mathrm{~Pa}$. Figures $2-4$ indicate that a strut imposes lower wall shear stress in its vicinity. The current model is a simple representation of single unit of the entire stent. Despite differences observed for the shape of the luminal surface between stented arteries with $40 \%$ and $80 \%$ stenoses, as seen in Figures 4(b) and 4(c), only a small discrepancy is observed for the distribution of wall shear stress for the whole stenosis percentages (Figure 4(a)).

In order to compare the behavior of shear stress between the steady flow and the transient flow, the time dependent flow simulations are also performed. Figures 5(b) to 5(d) represent the histograms for the percentage of luminal surface area with the wall shear stress less than $0.5 \mathrm{~Pa}(A<$ $0.5 \mathrm{~Pa}$ ), in five specified time instants of the cardiac cycle shown in Figure 5(a). The threshold of $0.5 \mathrm{~Pa}$ indicates a critical wall shear stress value to consider a region prone to restenosis [1, 16, 27]. Figures 5(b), 5(c), and 5(d) display the area percentages with wall shear stress values below $0.5 \mathrm{~Pa}$ for different plaques with thin, medium, and thick fibrous caps. Results reveal that the areas corresponding to wall shear stress below the critical value of $0.5 \mathrm{~Pa}$, unlike the steady state flow, are dependent of the instants taken on the cardiac pulse shown in Figure 5(a). The value of wall shear stress increases with the inlet velocity, and simultaneously the luminal surface area below the critical wall shear stress declines. Figures 5(b), 5(c), and 5(d) show that $A<0.5 \mathrm{~Pa}$ decreases at the systole $(0.141 \mathrm{~s})$ in all stenosed arteries. During the accelerating phase (e.g., $0.081 \mathrm{~s}$ ), $A<0.5 \mathrm{~Pa}$ varies significantly with the fibrous cap thickness. It is maximized when the blood velocity reaches a minimum. These results are in agreement with the results reported by Balossino et al. [1]. Figure 5 also demonstrates that $A<0.5 \mathrm{~Pa}$ is higher for $40 \%$ stenosis than the other stenosis percentages, particularly in thinner fibrous cap. This may be explained by comparing the data of fibrous cap thickness given in Tables 1 and 2 for different degrees of stenoses before and after stenting. Although $80 \%$ stenosis obtains a thinner fibrous cap after stenting, the whole plaque remains thicker in this case. Hence, in addition to fibrous cap thickness, the thickness of necrotic core and fibrosis are critical in assessing the risk of restenosis.

3.2. von Mises Stress Distribution. Figures 6(a), 6(b), 6(c), and $6(\mathrm{~d})$ demonstrate the maximum von Mises stress for $40 \%, 60 \%$, and $80 \%$ stenosed coronary arteries through the fibrous cap, necrotic core, fibrosis, and arterial wall layers, respectively. Note that the distribution of von Mises stress is also shown for corresponding parts in Figure 6 by colored contours for only $60 \%$ stenosis with the medium thickness of fibrous cap (Table 1).

Figure 6 reveals that the von Mises stress is significantly elevated within the fibrous cap and fibrosis layers by the stent insertion, while it is less affected within the necrotic core and arterial wall layers. Figure 6 also displays the effect of the fibrous cap thickness on von Mises stress. Among all plaque layers and arterial wall, the maximum values of von Mises stress are observed in the thin fibrous cap. Higher stresses are predicted in layers of thinner fibrous cap, while the thicker fibrous cap appears to play a protective role by reducing the levels of stress within the next plaque layers and arterial tissue 


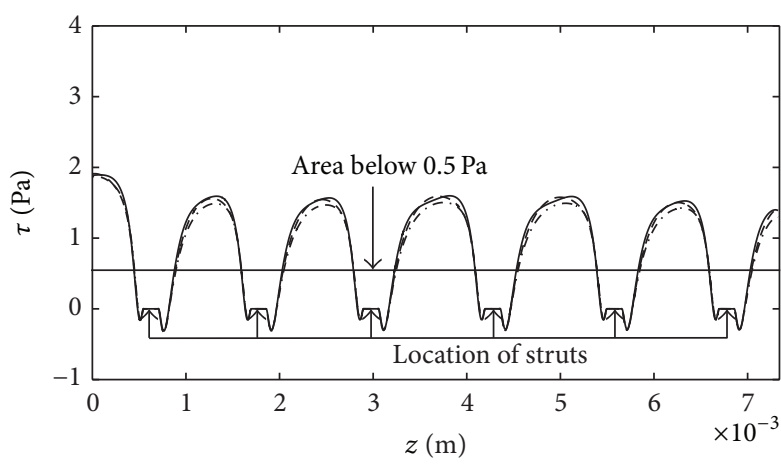

- - $40 \%$

-. - $60 \%$

$-80 \%$

(a)

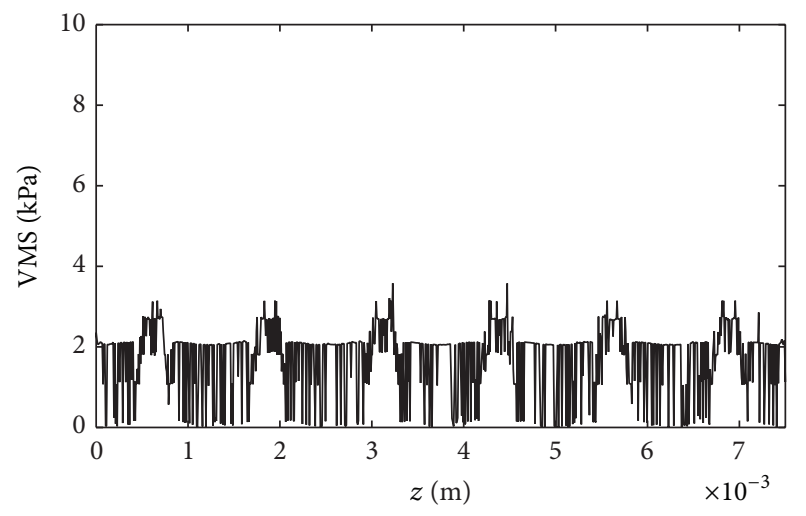

- FC-NC

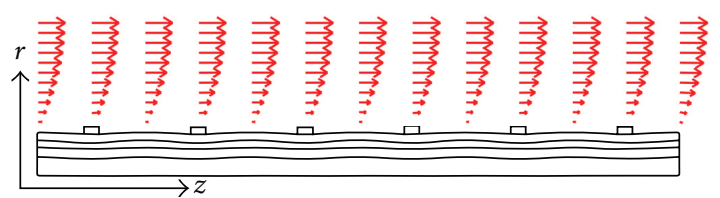

(b)

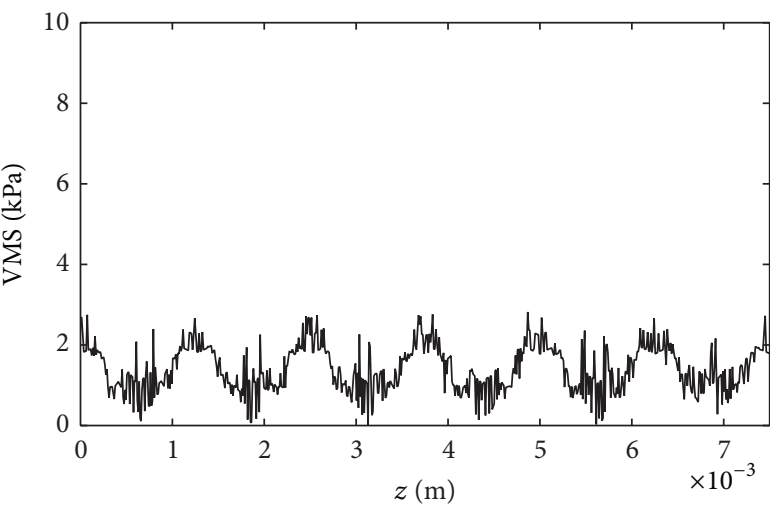

NC-F

(c)

(d)

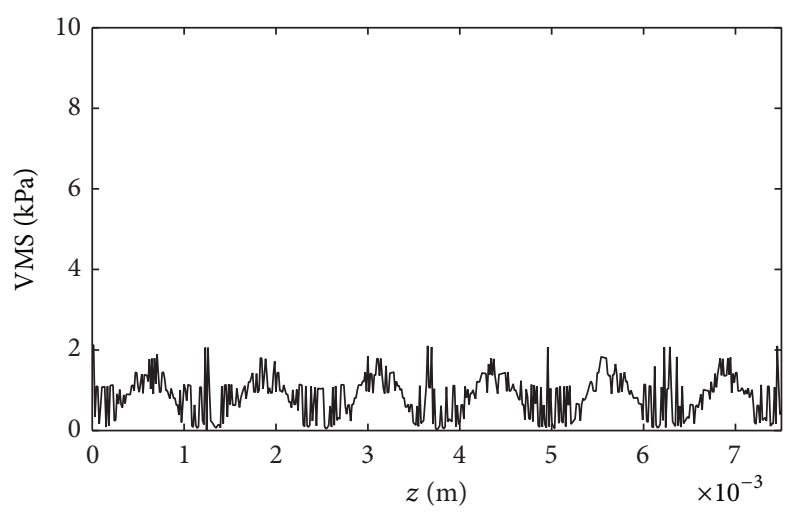

F-AW

(e)

Figure 2: (a) Wall shear stress distribution at the luminal side of the plaque surface in the stented arteries with multiple struts. The fibrous cap has the medium thickness. (b) Velocity profiles at the center of every two struts and on the middle of struts. (c)-(e) Distribution of von Mises stress along the interfaces of fibrous cap-necrotic core (FC-NC), necrotic core-fibrosis (NC-F), and fibrosis-arterial wall (F-AW) layers, respectively.

for a given inflation pressure [15]. Another interesting result observed in Figure 6 is the effect of the stenosis severity on the maximum von Mises stress through different layers. The maximum von Mises stress is observed within the fibrous cap in $80 \%$ stenosis that is significantly larger than those in $40 \%$ and $60 \%$ stenoses. Similar values have been calculated for $40 \%$ and $60 \%$ stenoses except at the thin fibrous cap that shows a lower value for $40 \%$ stenosis. These results are in 


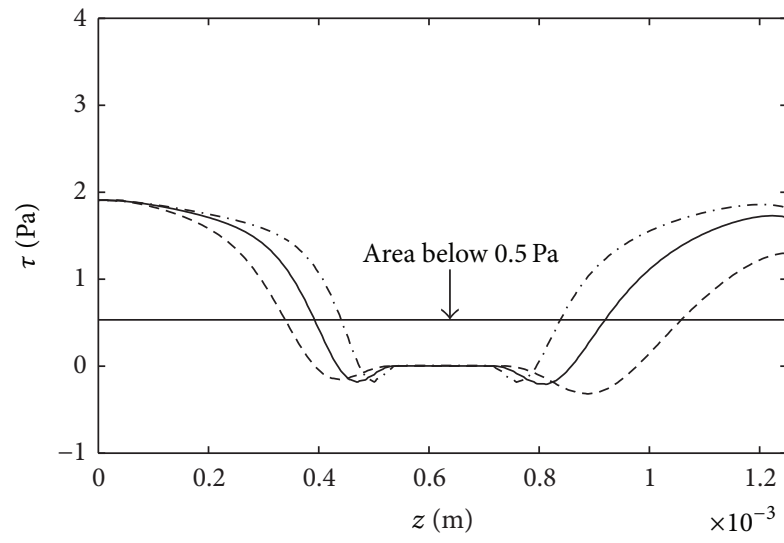

(a)

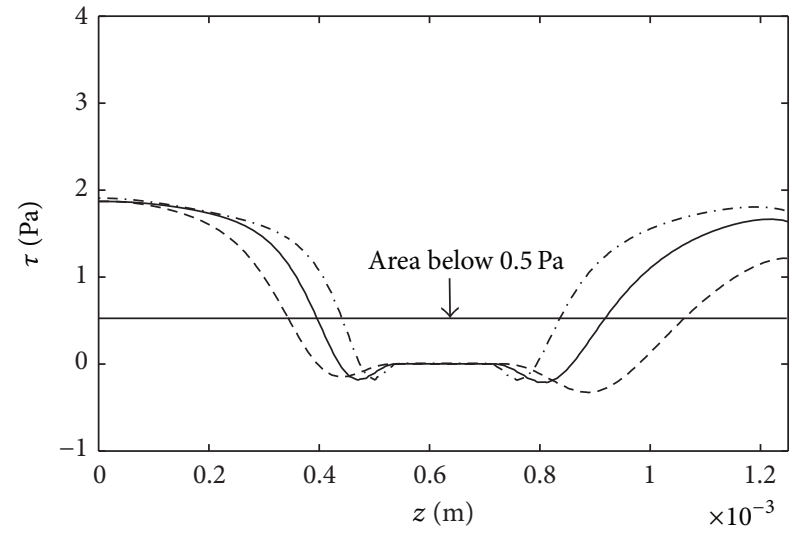

(b)

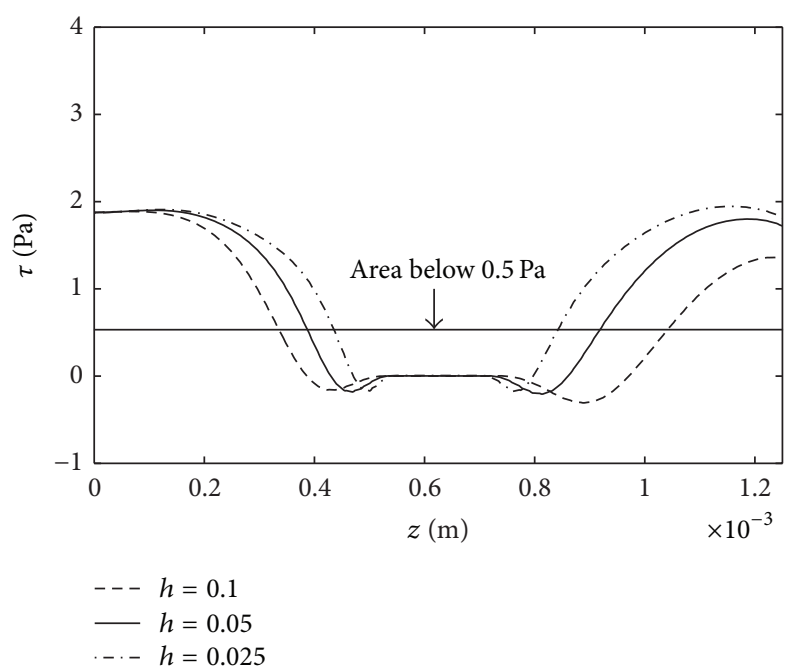

(c)

FIGURE 3: Wall shear stress distribution at the luminal side of the plaque surface with different magnitude of strut thickness $(h=0.1,0.05$, $0.025 \mathrm{~mm}$ ) for (a) $40 \%$, (b) $60 \%$, (c) $80 \%$ stenosed arteries. The fibrous cap has the medium thickness.

good agreement with the results reported by $\mathrm{Gu}$ et al. [21]. It should be mentioned that the maximum von Mises stress is observed in the fibrous cap layer in the vicinity of its contact to the strut corners. The presented values in Figure 6(a) are averaged within a neighborhood around the two struts corners of the radius of $20 \mu \mathrm{m}$, where the stress concentration occurs.

The maximum von Mises stress in the fibrous cap layer is one order of magnitude higher than that in other layers. The arterial wall displays the lowest values of von Mises stress. Also, these results reveal that in all layers excluding the fibrous cap layer, the maximum von Mises stress is higher in $40 \%$ stenosis than those in $60 \%$ and $80 \%$ stenoses. This can be associated with the effect of the thickness of different plaque layers in $40 \%$ stenosis that does not allow stress to be more uniformly distributed. Hence, locally elevated stresses are more likely except in the neighborhood of strut corners in fibrous cap thickness where the larger deformation in $80 \%$ stenosis imposes bigger stress concentration.
In addition to the opening of stenosed arteries by variable pressures mentioned in Table 2, simulations with the fixed opening pressure of $100 \mathrm{mmHg}$ are also performed. The main purpose of these simulations was to show how the stented coronary arteries display wall shear stress distribution differently due to discrepancies associated with the poststenting shape of the luminal surface and the lumen diameter. Figure 7(a) shows the wall shear stress distribution generated along the $z$-direction of the stented arteries with $40 \%, 60 \%$, and $80 \%$ stenoses. The fibrous cap has the medium thickness in Figure 7(a). Also, the straight line from $z=0.55 \mathrm{~mm}$ to $z=$ $0.7 \mathrm{~mm}$ represents the stent strut. Qualitatively, this graph is similar to the one shown in Figure 4(a). However, Figure 7(a) reveals the significant effect of the stenosis severity on wall shear stress distribution when the fixed opening pressure of $100 \mathrm{mmHg}$ is applied to open the artery. The wall shear stress magnitude also differs considerably between the two sides of the strut in $80 \%$ stenosis, which creates asymmetric hemodynamic conditions around the strut. It is due to large 


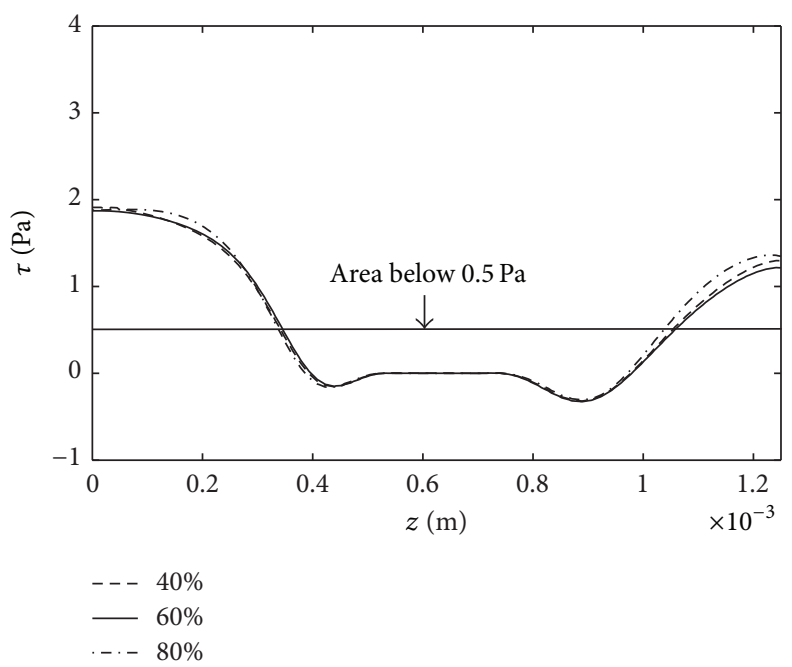

(a)

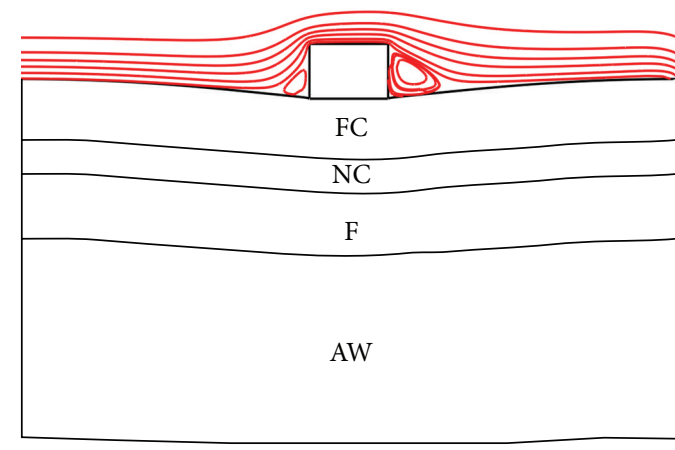

(b)

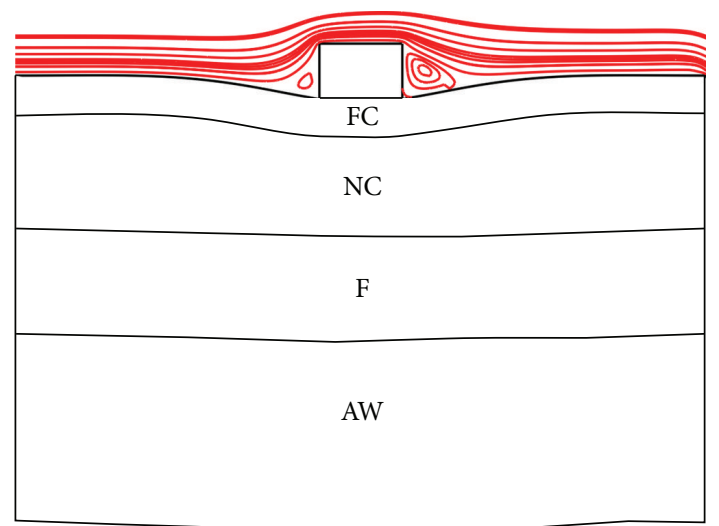

(c)

FIGURE 4: (a) Wall shear stress distribution at the luminal side of the plaque surface for $40 \%, 60 \%$, and $80 \%$ stenosed coronary arteries with medium thickness of fibrous cap. Deformed wall structure and blood flow velocity represented by streamlines in (b) $40 \%$ and (c) $80 \%$ stenoses.

recirculation zone created behind the strut due to small deformation of the wall. Figures 7(b) and 7(c) show the shape of stented arteries, the streamlines, and the displacement and thickness of plaque and wall layers around one strut, for $40 \%$ and $80 \%$ stenoses, respectively.

\section{Conclusions}

An axisymmetric model was employed to assess the hemodynamic and mechanical features in poststenting state of various stenosed coronary arteries. The risk level for injuries to the arterial wall due to stent replacement depends on many factors such as the stent design parameters, the opening pressure, the mechanical properties of the artery and plaque, and the geometry of the artery. The present study has focused on the influence of plaque severity and morphology on the hemodynamic stresses as well as the internal stresses created in the stented coronary artery. The computational model of stenosed coronary artery is composed of arterial wall (media layer) and a multilayer atherosclerotic plaque that can represent various stages of stenosis by the percentage of stenosis. The opening pressure is calculated for various stenoses to gain the diameter of healthy artery. Our simulations reveal that the wall shear stress distribution is independent of the stenoses rate if the healthy luminal diameter is reached after stenting. However, if the artery is not opened fully to its healthy diameter, the distribution of wall shear stress will depend on the stenoses rate and to the extent that the stenosed artery is expanded. To the best of our knowledge, this is the first study to take such an approach.

The plaque morphology, specifically the thickness of fibrous cap, has a significant influence on the stresses developed after stenting within the plaque layers and arterial wall. The thick fibrous cap layer protects the consequent layers by reduction of von Mises stress within the necrotic core, fibrosis, and arterial wall regardless of the stenosis size. However, the von Mises stress inside fibrous cap is highly dependent on the stenosis severity. Higher von Mises stress is observed in the fibrous cap layer corresponding to $80 \%$ stenosis. This suggests that higher pressures applied to $80 \%$ stenosis can induce a higher risk of vascular injury. The 

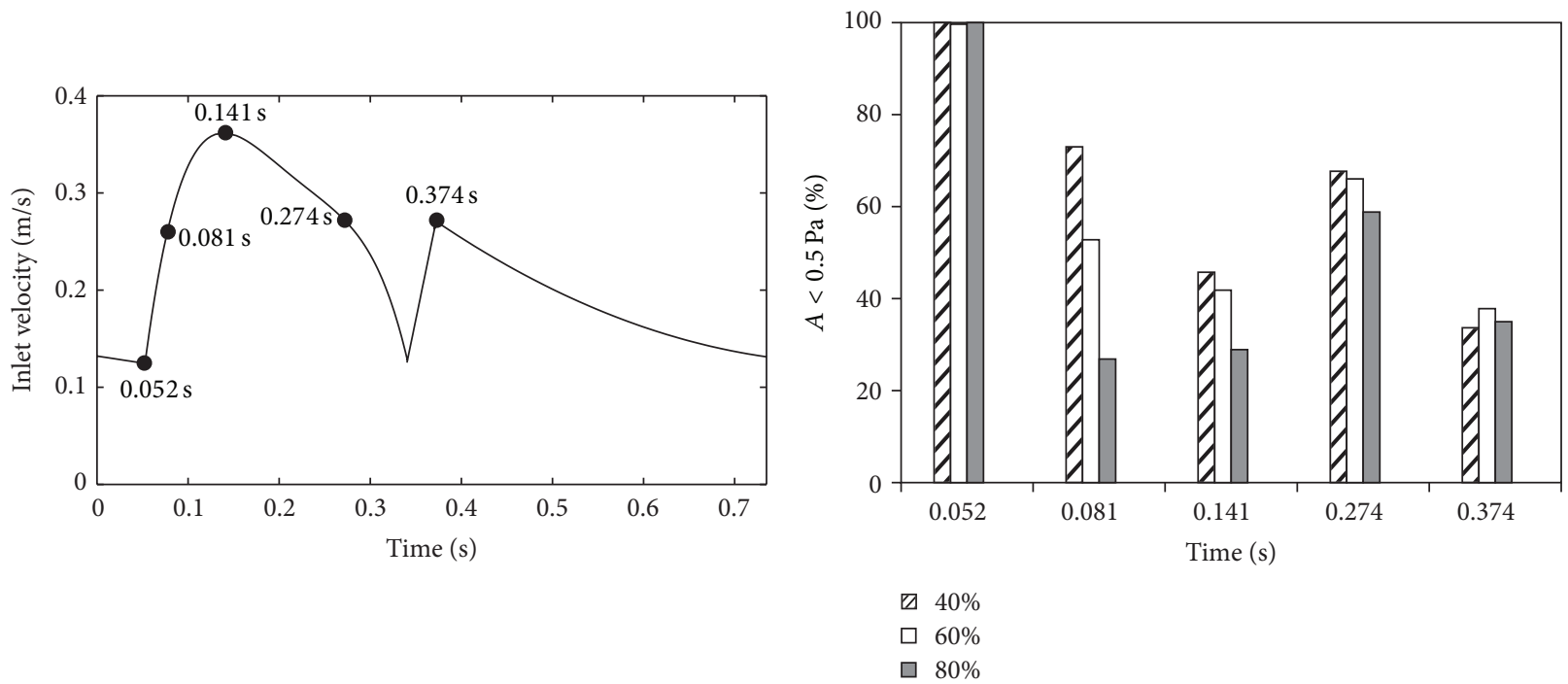

(a)
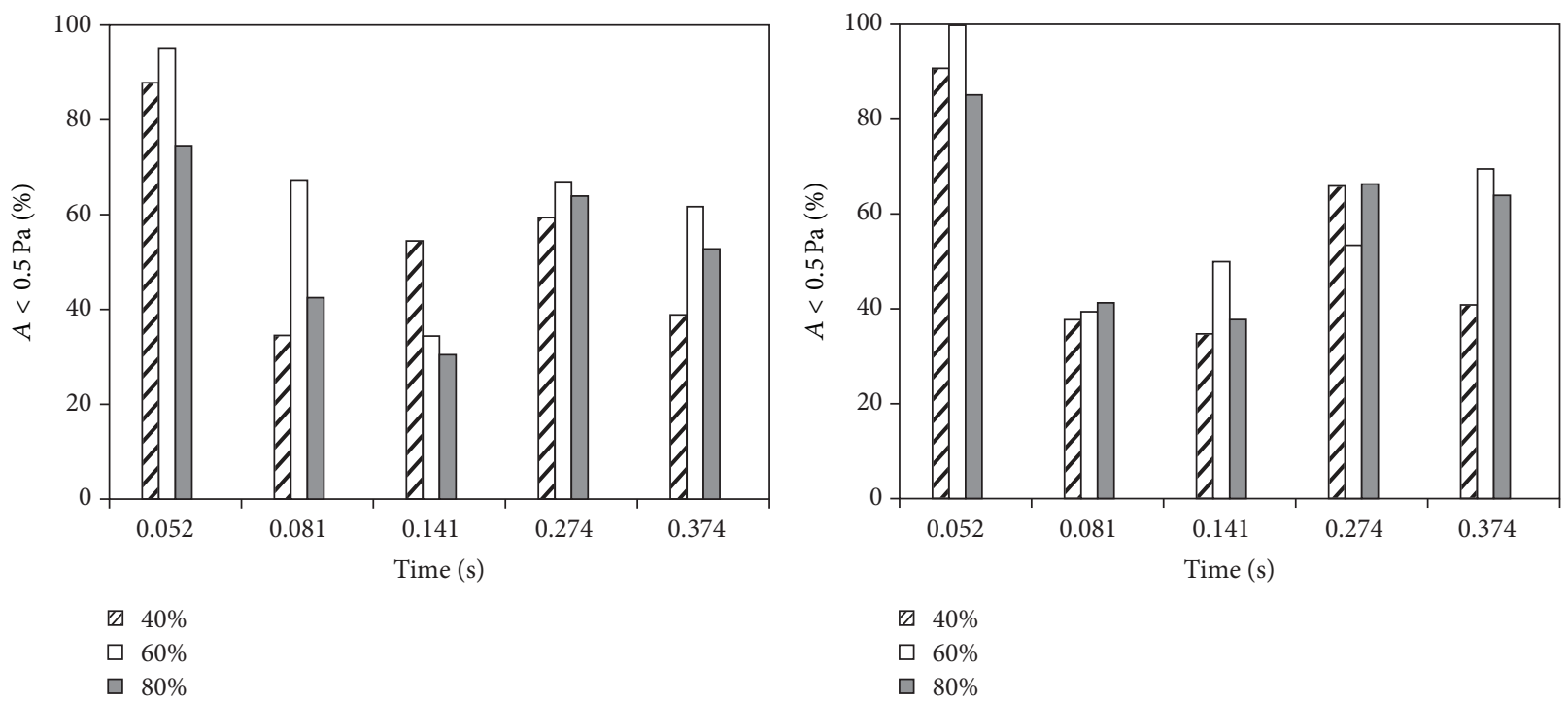

(c)

(d)

FIGURE 5: (a) Coronary inlet velocity pulse representing a cardiac cycle with five selected time instants marked on it. Histograms of the percentage of luminal wall area with wall shear stress values less than $0.5 \mathrm{~Pa}(A<0.5 \mathrm{~Pa})$ for $40 \%, 60 \%$, and $80 \%$ stenoses when fibrous cap is (b) thin, (c) medium, and (d) thick.

thinnest fibrous cap, in this study, is $27 \mu \mathrm{m}$ that belongs to the $80 \%$ stenosis.

The plaque morphology also affects the in-stent restenosis process, suggested by Pericevic et al. [15]. The fibrous cap thickness affects the wall shear stress in the region prone to restenosis. The time dependent flow simulation reveals that the value of wall shear stress increases with velocity, and simultaneously the luminal surface area below the critical wall shear stress declines. It reaches the maximum when the blood velocity is at the minimum. The lumen area with wall shear stress magnitude below $0.5 \mathrm{~Pa}$ is higher in the thinner fibrous caps of $40 \%$ stenosis, particularly at diastole. However, the prone area to restenosis is independent of stenosis severity as the fibrous cap thickens. For $80 \%$ stenosis, the lumen area with wall shear stress below $0.5 \mathrm{~Pa}$ is larger for thicker fibrous cap. The direct influence of the necrotic core volume is not discussed in the results. However, the growing necrotic core volume with the stenosis rate is considered.

The stent opening pressure affects the wall shear stress magnitude in poststenting state. Transient flow simulations reveal that the wall shear stress distribution significantly varies through different instants in cardiac cycle when high pressures applied. This study reveals that the risk of restenosis around the stent struts, where the wall shear stress is lowered, could remain similar in various rates of stenosis due to similar values of wall shear stress. However, the stress concentration 


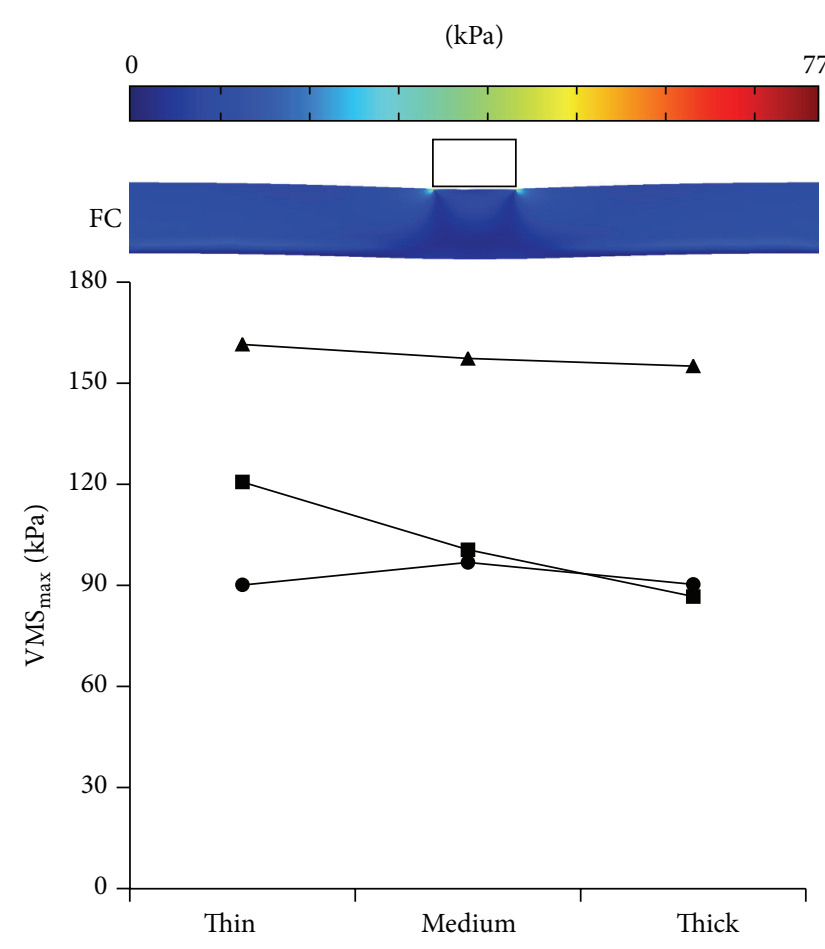

(a)

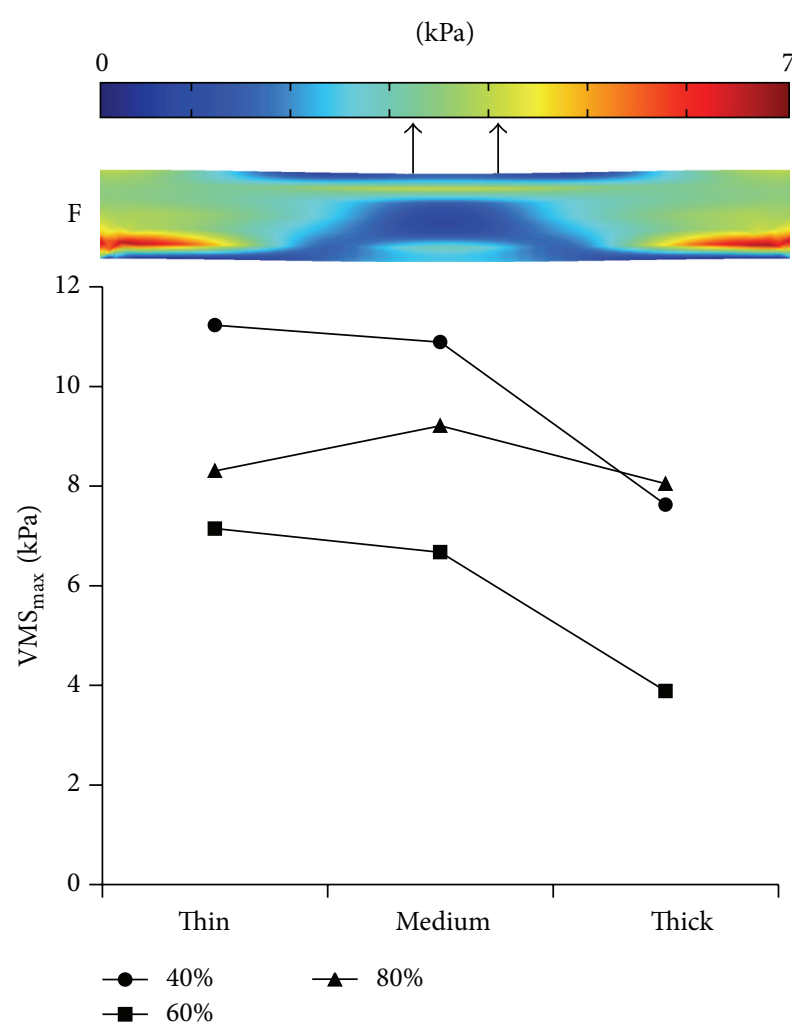

(c)

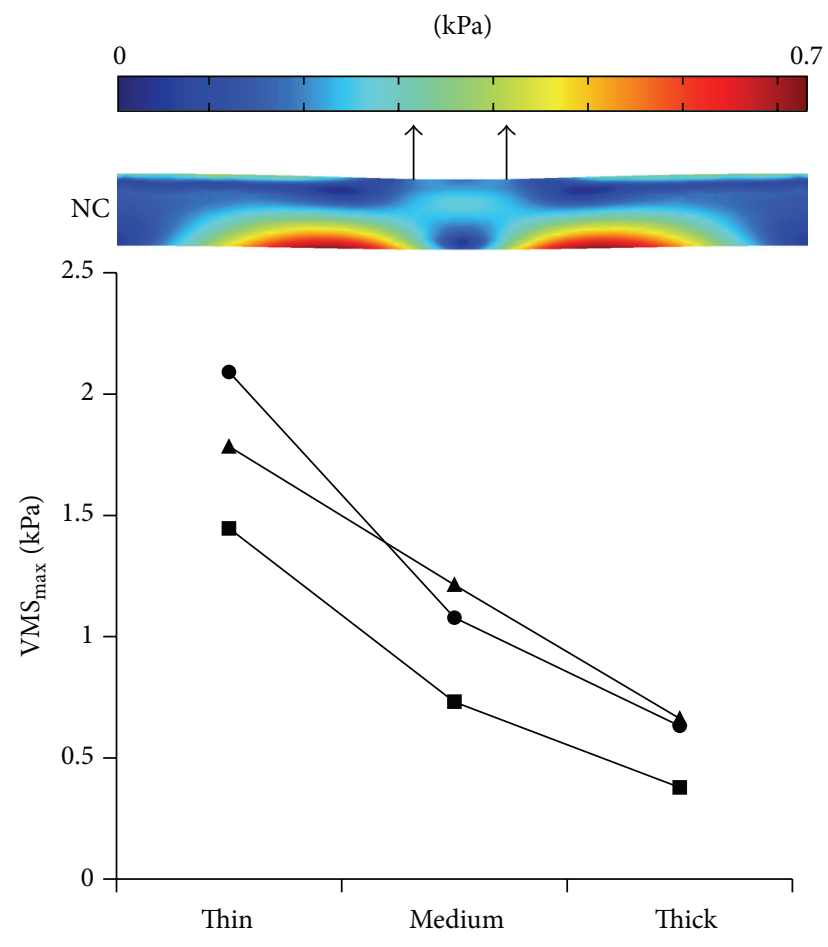

(b)

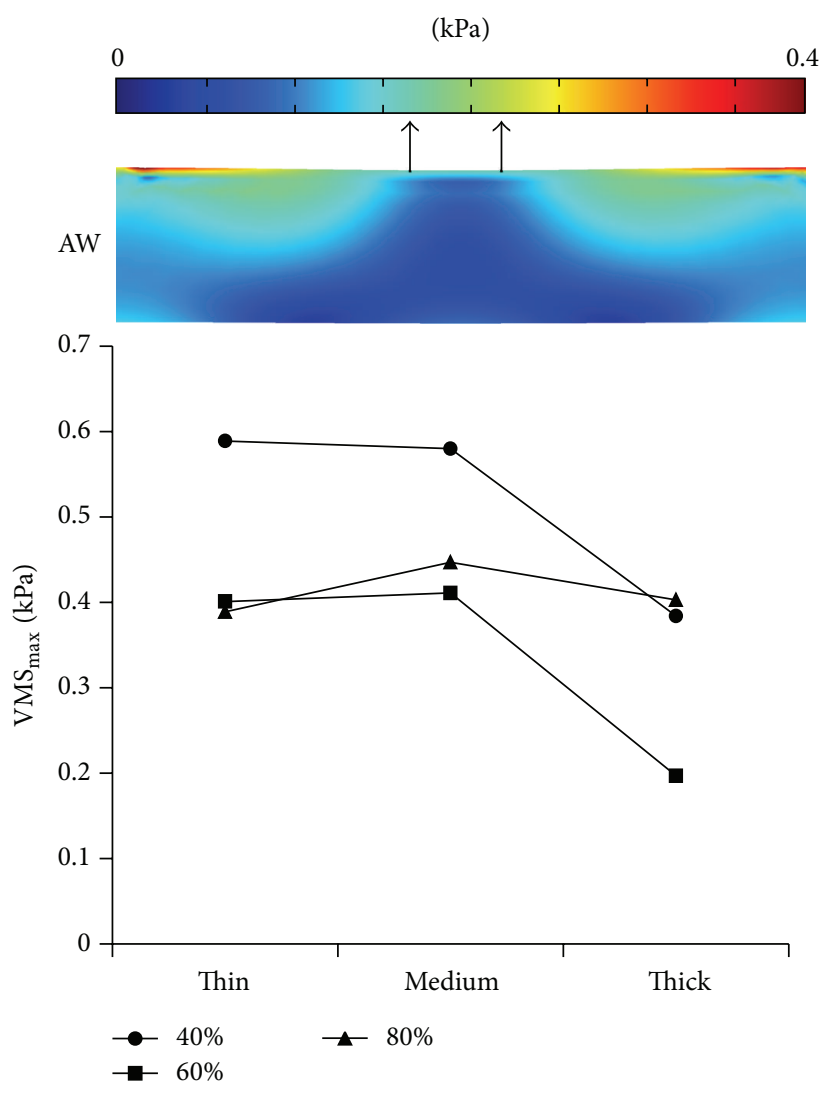

(d)

Figure 6: Maximum von Mises stress values against varying fibrous cap thickness (FCT) (thin, medium, thick) for $40 \%$, $60 \%$, and $80 \%$ stenosed coronaries, opened to a healthy lumen diameter of $2.68 \mathrm{~mm}$, on different layers. (a) Fibrous cap (FC) (area weighted average value of highly concentrated stress region), (b) necrotic core (NC), (c) fibrosis (F), and (d) arterial wall (AW). Contour plots represent the pattern of von Mises stress distribution through each layer. 


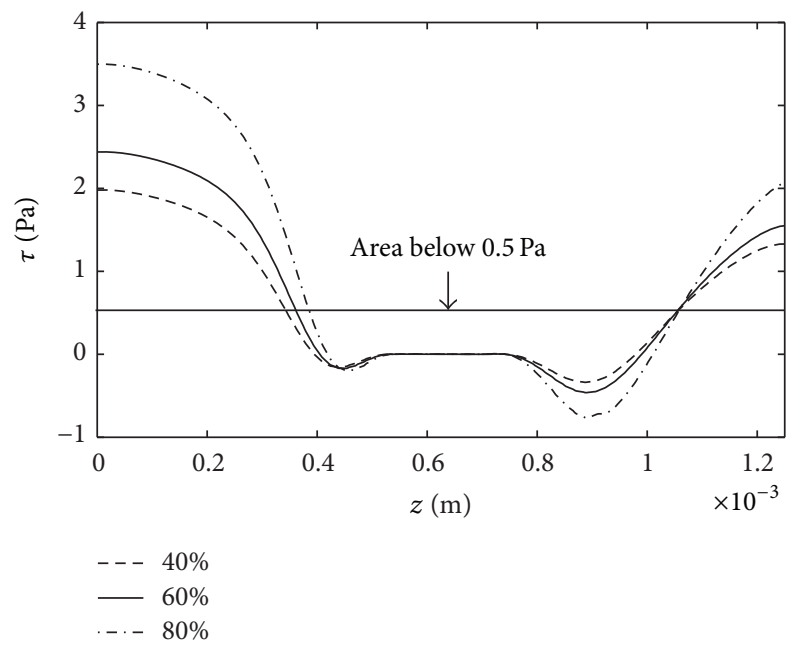

(a)

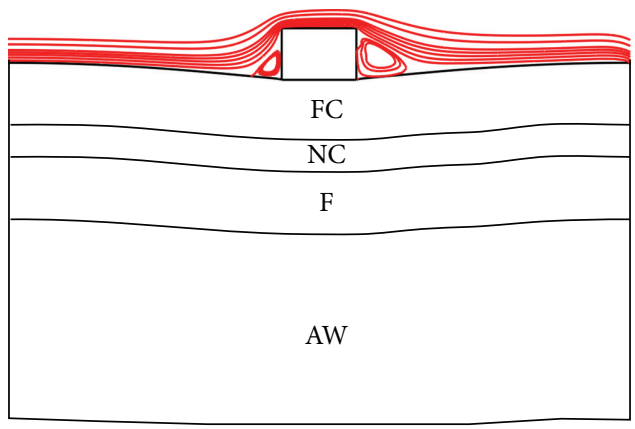

(b)

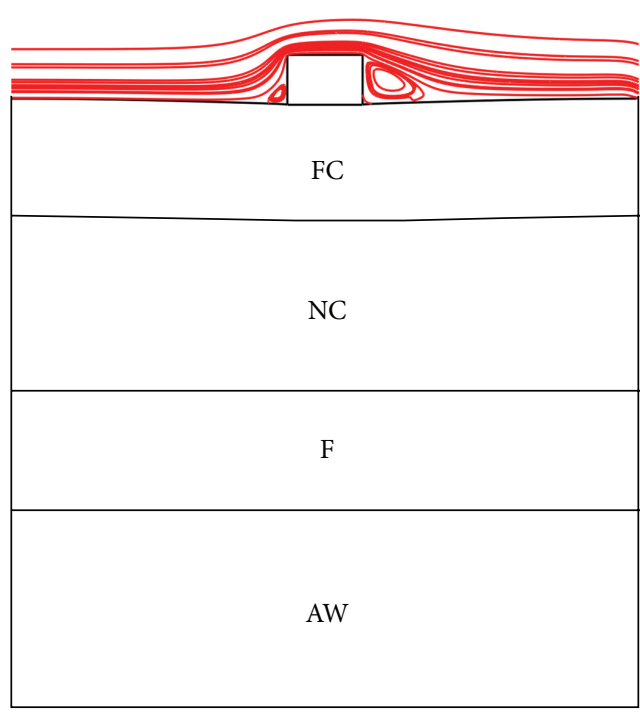

(c)

FIGURE 7: (a) Wall shear stress distribution at the luminal side of the plaque surface for $40 \%, 60 \%$, and $80 \%$ stenosed coronary arteries with medium thickness of fibrous cap. Deformed wall structure and blood flow velocity represented by streamlines in (b) $40 \%$ and (c) $80 \%$ stenoses. A fixed pressure of $100 \mathrm{mmHg}$ is applied to open the stenosed coronaries.

near the contact point of strut and the fibrous cap of the plaque rises notably in larger stenosis rates.

The thickness of stent struts influences significantly the rate of restenosis, independent of plaque severity. On the other hand, the interstrut distance affects the von Mises stress on plaque and arterial wall layers. Our results (not shown) reveal that the smaller interstrut distance enhances the prone region to restenosis particularly on downstream side of the strut. The present study is limited to the simplified and axisymmetric assumptions of plaque and artery. However, it simply describes the role of plaque structure, the thickness of stent struts, the severity of stenosis, and the flow conditions on the risk of restenosis and the level of von Mises stress created in poststenting stage. Future works will be devoted to study realistic stent models and linking them to the findings of the current model. Nevertheless, this type of simulation model provides useful and quick answers to improvements associated with the design of stents under various conditions of stenosis possibly identified in different patients.

\section{Conflict of Interests}

The authors declare that there is no conflict of interests regarding the publication of this paper.

\section{Acknowledgments}

The authors would like to acknowledge the supporting project by the Academy of Finland under Grant no. 123938. The first author also acknowledges the funding provided by the Finnish Graduate School in Computational Fluid Dynamics. 


\section{References}

[1] R. Balossino, F. Gervaso, F. Migliavacca, and G. Dubini, "Effects of different stent designs on local hemodynamics in stented arteries," Journal of Biomechanics, vol. 41, no. 5, pp. 1053-1061, 2008.

[2] F. Migliavacca, F. Gervaso, M. Prosi et al., "Expansion and drug elution model of a coronary stent," Computer Methods in Biomechanics and Biomedical Engineering, vol. 10, no. 1, pp. 6373, 2007.

[3] C. Rogers and E. R. Edelman, "Endovascular stent design dictates experimental restenosis and thrombosis," Circulation, vol. 91, no. 12, pp. 2995-3001, 1995.

[4] P. J. Prendergast, C. Lally, S. Daly et al., "Analysis of prolapse in cardiovascular stents: a constitutive equation for vascular tissue and finite-element modelling," Journal of Biomechanical Engineering, Transactions of the ASME, vol. 125, no. 5, pp. 692699, 2003.

[5] R. Tominaga, H. E. Kambic, H. Emoto, H. Harasaki, C. Sutton, and J. Hollman, "Effects of design geometry of intravascular endoprostheses on stenosis rate in normal rabbits," American Heart Journal, vol. 123, no. 1, pp. 21-28, 1992.

[6] C. Lally, F. Dolan, and P. J. Prendergast, "Cardiovascular stent design and vessel stresses: a finite element analysis," Journal of Biomechanics, vol. 38, no. 8, pp. 1574-1581, 2005.

[7] G. A. Holzapfel, T. C. Gasser, and M. Stadler, "A structural model for the viscoelastic behavior of arterial walls: continuum formulation and finite element analysis," European Journal of Mechanics, A/Solids, vol. 21, no. 3, pp. 441-463, 2002.

[8] J. E. Moore Jr., C. Xu, S. Glagov, C. K. Zarins, and D. N. $\mathrm{Ku}$, "Fluid wall shear stress measurements in a model of the human abdominal aorta: oscillatory behavior and relationship to atherosclerosis," Atherosclerosis, vol. 110, no. 2, pp. 225-240, 1994.

[9] J. M. Jiménez and P. F. Davies, "Hemodynamically driven stent strut design," Annals of Biomedical Engineering, vol. 37, no. 8, pp. 1483-1494, 2009.

[10] J. F. LaDisa Jr., D. A. Hettrick, L. E. Olson et al., "Stent implantation alters coronary artery hemodynamics and wall shear stress during maximal vasodilation," Journal of Applied Physiology, vol. 93, no. 6, pp. 1939-1946, 2002.

[11] J. Mejia, B. Ruzzeh, R. Mongrain, R. Leask, and O. F. Bertrand, "Evaluation of the effect of stent strut profile on shear stress distribution using statistical moments," BioMedical Engineering Online, vol. 8, article 8, 2009.

[12] D. Tang, C. Yang, J. Zheng et al., "Quantifying effects of plaque structure and material properties on stress distributions in human atherosclerotic plaques using 3D FSI models," Journal of Biomechanical Engineering, vol. 127, no. 7, pp. 1185-1194, 2005.

[13] H. Gao and Q. Long, "Effects of varied lipid core volume and fibrous cap thickness on stress distribution in carotid arterial plaques," Journal of Biomechanics, vol. 41, no. 14, pp. 3053-3059, 2008.

[14] J. Ohayon, G. Finet, A. M. Gharib et al., "Necrotic core thickness and positive arterial remodeling index: emergent biomechanical factors for evaluating the risk of plaque rupture," The American Journal of Physiology-Heart and Circulatory Physiology, vol. 295, no. 2, pp. H717-H727, 2008.

[15] I. Pericevic, C. Lally, D. Toner, and D. J. Kelly, "The influence of plaque composition on underlying arterial wall stress during stent expansion: the case for lesion-specific stents," Medical Engineering and Physics, vol. 31, no. 4, pp. 428-433, 2009.
[16] M. Gay and L. T. Zhang, "Numerical studies on fluid-structure interactions of stent deployment and stented arteries," International Journal for Numerical Methods in Fluids, vol. 25, no. 1, pp. 61-72, 2009.

[17] H. Zahedmanesh and C. Lally, "Determination of the influence of stent strut thickness using the finite element method: implications for vascular injury and in-stent restenosis," Medical and Biological Engineering and Computing, vol. 47, no. 4, pp. 385393, 2009.

[18] S. N. D. Chua, B. J. MacDonald, and M. S. J. Hashmi, "Effects of varying slotted tube (stent) geometry on its expansion behaviour using finite element method," Journal of Materials Processing Technology, vol. 155-156, no. 1-3, pp. 1764-1771, 2004.

[19] H. C. Stary, A. B. Chandler, R. E. Dinsmore et al., "A definition of advanced types of atherosclerotic lesions and a histological classification of atherosclerosis," Circulation, vol. 92, no. 5, pp. 1355-1374, 1995.

[20] R. Virmani, A. P. Burke, A. Farb, and F. D. Kolodgie, "Pathology of the vulnerable plaque," Journal of the American College of Cardiology, vol. 47, no. 8, pp. C13-C18, 2006.

[21] L. Gu, S. Zhao, A. K. Muttyam, and J. M. Hammel, “The relation between the arterial stress and restenosis rate after coronary stenting," Journal of Medical Devices, Transactions of the ASME, vol. 4, no. 3, Article ID 031005, 2010.

[22] J. Tambaca, S. Canic, M. Kosor, R. D. Fish, and D. Paniagua, "Mechanical behavior of fully expanded commercially available endovascular coronary stents," Texas Heart Institute Journal, vol. 38, no. 5, pp. 491-501, 2011.

[23] S. Čanić, C. J. Hartley, D. Rosenstrauch, J. Tambača, G. Guidoboni, and A. Mikelić, "Blood flow in compliant arteries: an effective viscoelastic reduced model, numerics, and experimental validation," Annals of Biomedical Engineering, vol. 34, no. 4, pp. 575-592, 2006.

[24] S. Balocco, O. Basset, G. Courbebaisse et al., "Estimation of the viscoelastic properties of vessel walls using a computational model and Doppler ultrasound," Physics in Medicine and Biology, vol. 55, no. 12, pp. 3557-3575, 2010.

[25] R. A. Baldewsing, C. L. de Korte, J. A. Schaar, F. Mastik, and A. F. W. van der Steen, "A finite element model for performing intravascular ultrasound elastography of human atherosclerotic coronary arteries," Ultrasound in Medicine and Biology, vol. 30, no. 6, pp. 803-813, 2004.

[26] S. Le Floc'h, G. Cloutier, G. Finet, P. Tracqui, R. I. Pettigrew, and J. Ohayon, "On the potential of a new IVUS elasticity modulus imaging approach for detecting vulnerable atherosclerotic coronary plaques: in vitro vessel phantom study," Physics in Medicine and Biology, vol. 55, no. 19, pp. 5701-5721, 2010.

[27] H. J. Kim, I. E. Vignon-Clementel, J. S. Coogan, C. A. Figueroa, K. E. Jansen, and C. A. Taylor, "Patient-specific modeling of blood flow and pressure in human coronary arteries," Annals of Biomedical Engineering, vol. 38, no. 10, pp. 3195-3209, 2010.

[28] A. Brandts, S. D. Roes, J. Doornbos et al., "Right coronary artery flow velocity and volume assessment with spiral K-space sampled breathhold velocity-encoded MRI at 3 Tesla: accuracy and reproducibility," Journal of Magnetic Resonance Imaging, vol. 31, no. 5, pp. 1215-1223, 2010.

[29] J. Jung, R. W. Lyczkowski, C. B. Panchal, and A. Hassanein, "Multiphase hemodynamic simulation of pulsatile flow in a coronary artery," Journal of Biomechanics, vol. 39, no. 11, pp. 2064-2073, 2006. 


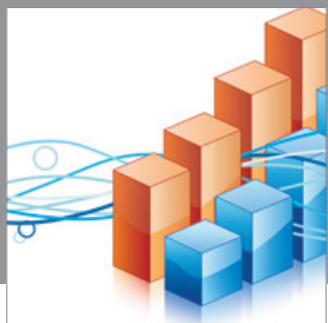

Advances in

Operations Research

mansans

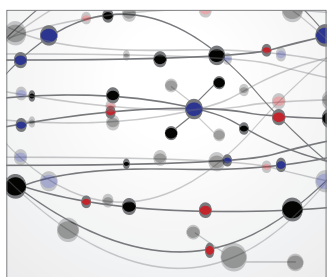

The Scientific World Journal
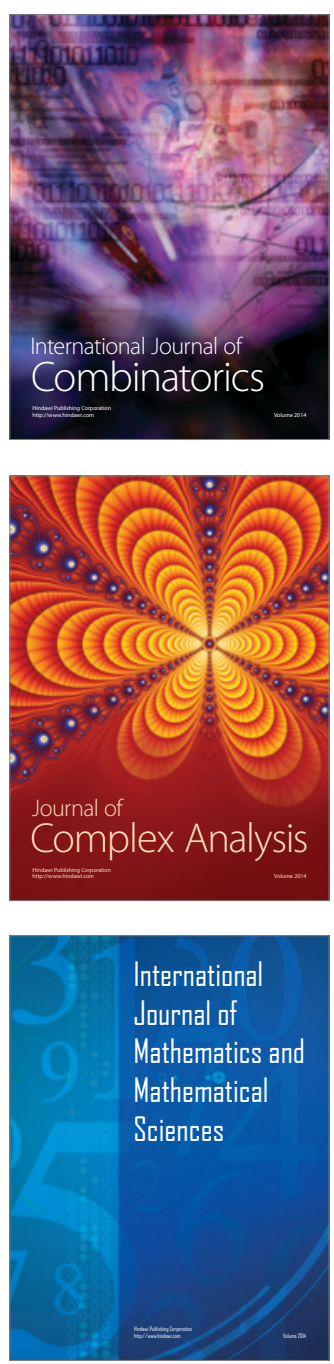
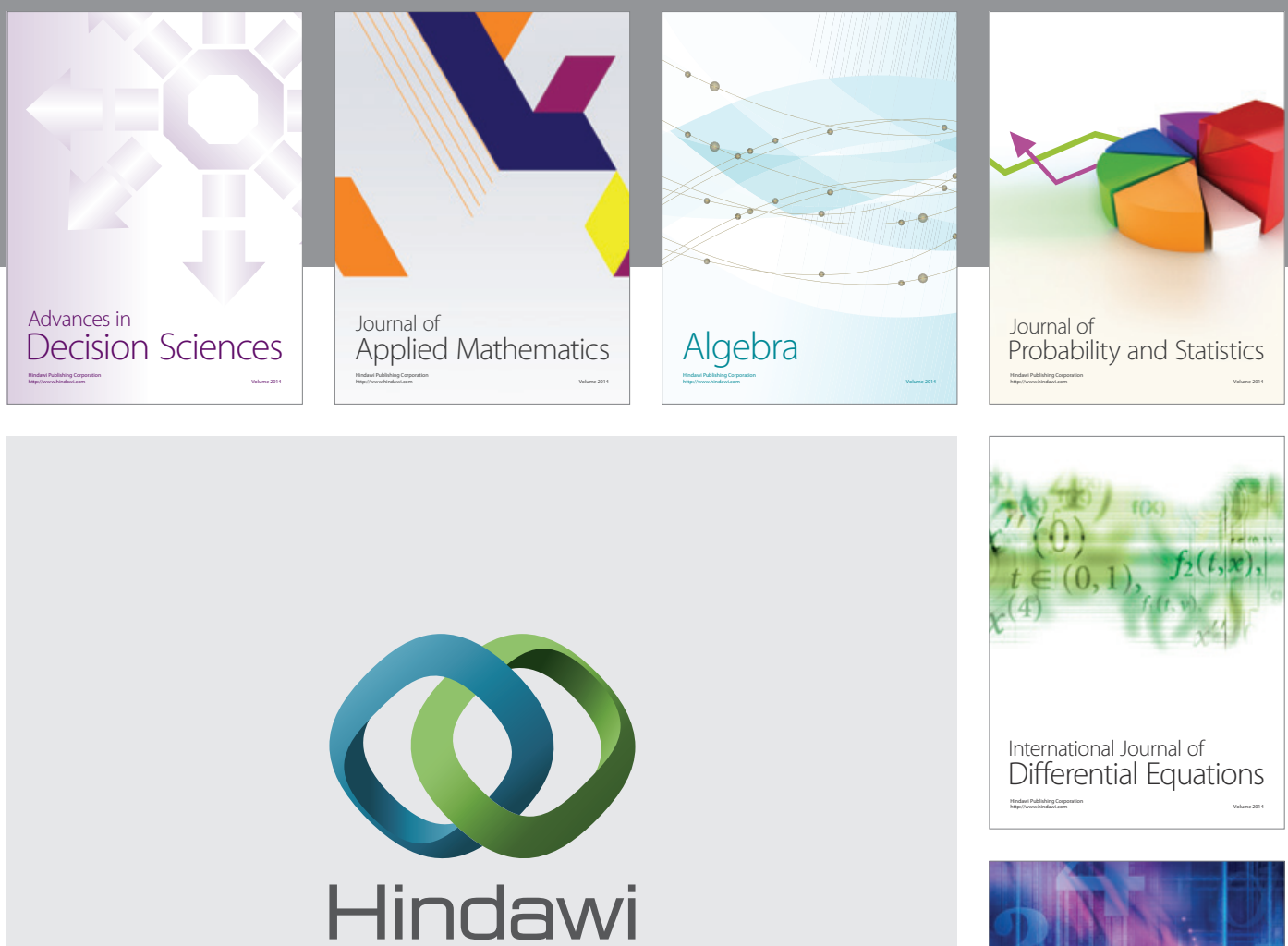

Submit your manuscripts at http://www.hindawi.com
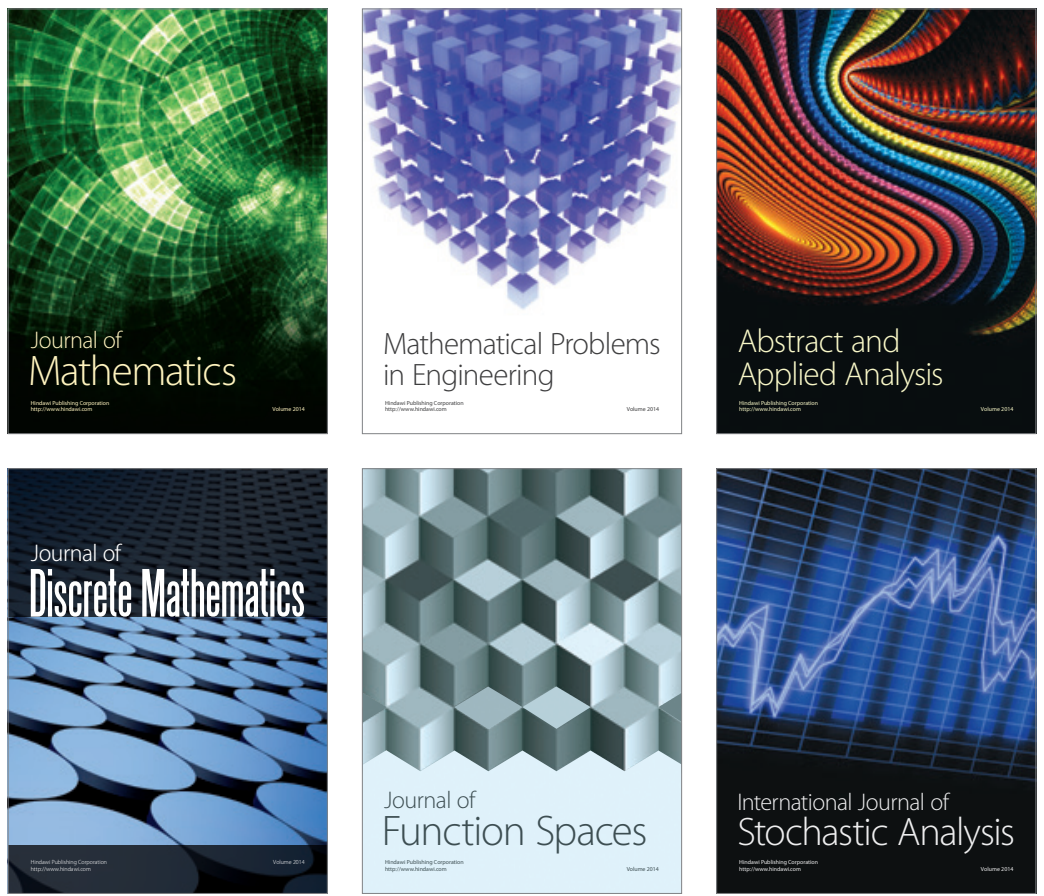

Journal of

Function Spaces

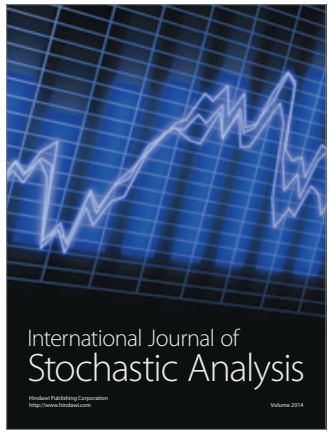

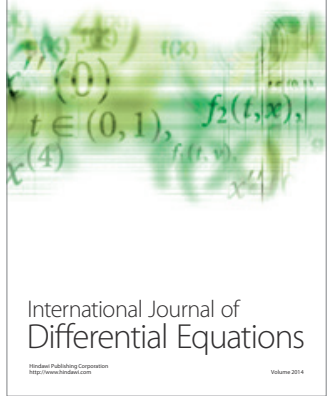
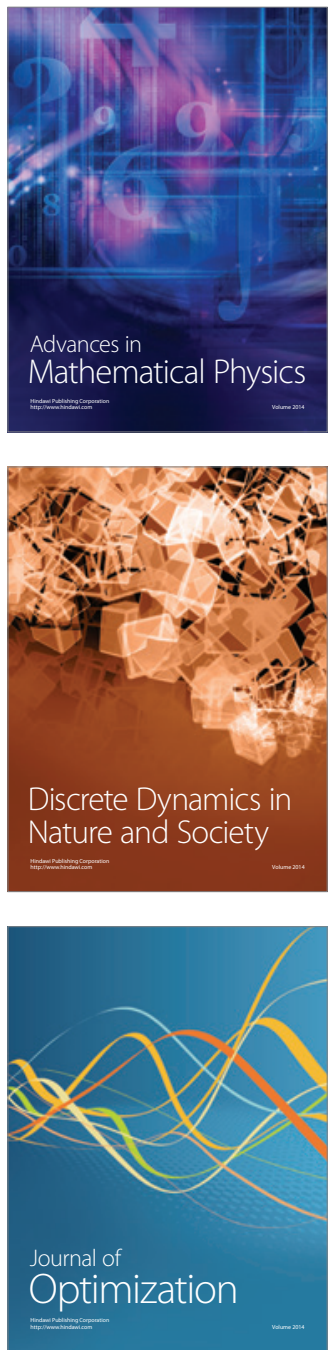